Disponível em

http://www.anpad.org.br/rac

RAC, Rio de Janeiro, v. 19, n. 3, art. 6, pp. 398-418, Maio/Jun. 2015

http://dx.doi.org/10.1590/1982-7849rac20151711

$($ (c) EY-No

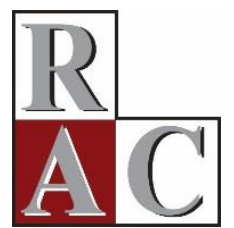

\title{
Estágios do Ciclo de Vida e Qualidade das Informações Contábeis no Brasil
}

\author{
Life Cycle Stages and Earnings Quality in Brazil
}

Ailza Silva de Lima

Universidade Federal da Paraíba - UFPB

Evelyne Vilhete Antonio de Carvalho Universidade Federal da Paraíba - UFPB

Edilson Paulo

Universidade Federal da Paraíba - UFPB

Luiz Felipe de Araújo Pontes Girão

Universidade Federal da Paraíba - UFPB

Artigo recebido em 04.11.2013. Última versão recebida em 06.02.2015. Aprovado em 08.02.2015. 


\title{
Resumo
}

O objetivo desse estudo foi analisar o efeito dos estágios de ciclo de vida (ECD) das empresas listadas na BM\&FBovespa no que tange à qualidade das suas informações contábeis (QIC), entre 1995 e 2011. Após a classificação das empresas de acordo com seus ECD, foi medida a QIC por meio do conservadorismo, da persistência dos resultados e fluxos de caixa, e do gerenciamento dos resultados. Os resultados da pesquisa sugerem que existem diferenças significativas na qualidade das informações contábeis, exceto para o gerenciamento de resultados contábeis, entre os estágios de ciclo de vida das companhias abertas brasileiras.

Palavras-chave: ciclo de vida; conservadorismo; gerenciamento de resultados; persistência; qualidade das informações contábeis.

\begin{abstract}
The aim of this study was to analyze the effect of the life-cycle stages (LCS) of companies listed on BM\&FBovespa regarding the quality of accounting information (AIQ), from 1995 to 2011. After companies were classified according to their LCS, AIQ were measured by conservatism, earnings and cash flows persistence, and earnings management. Our results suggest that there are significant differences in accounting quality, except for earnings management, in Brazilian companies' life-cycle stages.
\end{abstract}

Key words: life-cycle; conservatism; earnings management; persistence; earnings quality. 


\section{Introdução}

Embora os relatórios contábeis sejam padronizados e todas as empresas estejam obrigadas a divulgar uma determinada quantidade de informações sobre os seus resultados operacionais e condições financeiras, firmas do mesmo setor e com características econômicas e financeiras semelhantes, e que divulgam a mesma quantidade de informações, não são avaliadas igualmente. $\mathrm{O}$ valor de mercado de uma empresa pode ser influenciado por diferentes atributos econômicos, tais como as funções de produção, oferta e demanda ou o conjunto de oportunidades de investimentos e risco. Outros fatores podem justificar a diferenciação de valor de mercado entre empresas similares, nas quais se destacam os estágios de ciclo de vida da firma (Liu, 2008; Park \& Chen, 2006; Xu, 2007).

Os estágios de ciclo de vida de uma firma podem exercer efeitos significativos sobre os aspectos mercadológicos, estratégias de investimentos e financiamentos em cada fase de vida da empresa. Drake (2013), por exemplo, evidencia que variações nas receitas de venda, retorno sobre ativo, fluxos de caixa e persistência dos lucros são diferentes entre os estágios do ciclo de vida da firma. Por outro lado, as pesquisas têm evidenciado a relevância do conteúdo informacional dos relatórios contábeis no processo de avaliação de desempenho dos gestores e da empresa, bem como na precificação das firmas (Dechow, Ge, \& Schrand, 2010; Richardson, Tuna, \& Wysocki, 2010).

Apesar de as informações contábeis serem reguladas pelos órgãos responsáveis pelo funcionamento dos mercados, a literatura também contempla que os gestores podem fazer escolhas contábeis de forma a atingir algum objetivo específico, afetando, assim, a qualidade de informação contábil (Dechow et al., 2010; Xu, 2007). Dessa forma, além da discricionariedade dos gestores, existe a possibilidade de que o ciclo de vida da firma afete a qualidade dos números reportados por elas e, consequentemente, a avaliação de seu valor. O conservadorismo contábil, por exemplo, influencia a avaliação das firmas, sendo que empresas em diferentes estágios de ciclo de vida têm diferentes características financeiras que afetam o conservadorismo e, consequentemente, o value relevance da informação contábil (Park \& Chen, 2006).

Algumas pesquisas (E. L. Black, 1998; Ou \& Penman, 1989; Park \& Chen, 2006; Penman \& Zhang, 2002; Xu, 2007) estudaram o efeito do conservadorismo sobre o valor da empresa em diferentes momentos do ciclo de vida. Estes trabalhos evidenciaram que firmas em estágio de crescimento geralmente optam por mensurar seu desempenho de forma menos conservadora do que empresas em outras fases (maturidade e declínio), e que as empresas em declínio usam práticas contábeis mais conservadoras do que as empresas em estágio de maturidade. Além disso, segundo os mesmos autores, as empresas em crescimento têm uma contabilidade menos conservadora, pois necessitam atrair os investidores através da promessa de altas taxas de retorno. Destaca-se, também, o trabalho de Dechow e Ge (2006), que constataram que empresas maduras e em declínio tendem a gerenciar os seus resultados para baixo, evitando o pagamento de altos dividendos.

No entanto, observa-se que ainda existe uma lacuna na literatura que trata da relação entre a qualidade dos números contábeis reportados e o ciclo de vida das empresas. No que se refere às pesquisas nacionais, o tema é praticamente inexplorado. Além do mais, os trabalhos internacionais analisam, isoladamente, o comportamento de um dos atributos da qualidade da informação contábil. Sendo assim, essa pesquisa busca contribuir com a discussão nacional sobre qualidade das informações contábeis, com a inclusão dos estágios do ciclo de vida como um dos determinantes da qualidade da informação contábil, bem como com o debate internacional sobre a relação entre a qualidade da informação contábil e os estágios do ciclo de vida, com a inclusão dos diversos atributos da qualidade dos lucros, como persistência, conservadorismo e gerenciamento de resultados (Dechow et al., 2010).

Considerando o exposto nos parágrafos anteriores, o presente estudo tem o seguinte problema de pesquisa: De que forma os diferentes estágios do ciclo de vida da firma afetam a qualidade das informações contábeis reportadas pelas companhias abertas brasileiras? 
O objetivo geral desta pesquisa foi analisar o comportamento da qualidade das informações contábeis nos diferentes estágios de ciclo de vida das companhias listadas na BM\&FBovespa.

O desenvolvimento deste trabalho se justifica pelo fato de que: (a) estudos anteriores constataram que a qualidade de informação contábil exerce papel importante na avaliação do desempenho econômico e no processo de valoração da empresa (Ahmed, Morton, \& Schaefer, 2000; Anthony \& Ramesh, 1992; Dechow \& Ge, 2006; Drake, 2013; Penman \& Zhang, 2002); (b) as pesquisas anteriores demonstram que o estágio de ciclo de vida afeta o conteúdo informacional da contabilidade, independentemente de comportamento idiossincrático dos gestores, porém somente analisam uma única característica da informação contábil isoladamente; (c) o mercado de capitais brasileiro possui idiossincrasias que divergem dos ambientes explorados nas pesquisas anteriores; (d) inexistência de trabalhos no ambiente brasileiro que versem sobre o comportamento dos números contábeis nos diferentes estágios do ciclo de vida da firma, sendo que estudos sobre a qualidade dos números contábeis em mercados emergentes tornam-se oportunos, pois os investidores nestes ambientes possuem um conjunto de informações limitadas, comparativamente aos mercados mais desenvolvidos (Alali \& Foote, 2012), elevados benefícios privados de controle, sistema de governança e enforcement historicamente fracos (B. S. Black, Carvalho, \& Gorga, 2012).

Assim, este estudo busca contribuir com a compreensão dos efeitos dos diferentes estágios de ciclo de vida das empresas na qualidade das informações contábeis no ambiente brasileiro, através da investigação do comportamento do conservadorismo, persistência e gerenciamento de resultados em diferentes estágios de ciclo de vida da firma, na qual apresenta evidências de que existe o efeito dos estágios dos ciclos de vida das empresas na qualidade da informação contábil. Essas evidências podem auxiliar os investidores e os órgãos reguladores no processo de avaliação e monitoramento das companhias.

\section{Fundamentação Teórica}

\section{Ciclo de vida da firma}

De acordo com Samadiyan e Rezaei (2012), um dos modelos mais utilizados para análise do valor da empresa e do seu posicionamento no mercado é o do ciclo de vida. Drake (2013) descreve que o objetivo da análise do ciclo de vida da firma é avaliar como as variações nos incentivos, restrições e estratégias ao longo do ciclo de vida estão relacionadas com as decisões dos gestores e o desempenho da empresa.

De modo geral, a empresa, ao nascer, não possui informação significativa sobre o mercado, como também não possui informações suficientes sobre o seu processo operacional (fornecedores e clientes). No estágio inicial, por não ter experiência, a empresa normalmente não possui maiores habilidades no processo produtivo. Entretanto, esse cenário muda à medida que ocorre o amadurecimento da sua relação com o processo operacional, seus fornecedores e clientes passam a ser conhecidos, melhorando o processo de comercialização. Adquire-se agilidade ao produzir, minimizando os custos e levando-a ao crescimento (Marshall, 1890).

Mueller (1972) considera que a empresa típica exibirá um padrão de crescimento com um período lento no início da sua entrada no mercado, seguindo um período de rápido crescimento, eventualmente, até à maturidade, depois à estagnação ou ao crescimento lento. Essas fases do ciclo de vida são definidas como a configuração de variáveis relacionadas ao contexto econômico em que a organização está inserida, bem como com a sua estrutura organizacional. Com base nisso, as dimensões contextuais são definidas como aquelas compostas pela idade da organização, por seu tamanho e taxa de crescimento, fazendo-se uma relação com os desafios enfrentados pela empresa. Por outro lado, a estrutura organizacional é definida como a formalização, centralização e distinções do nível organizacional (Mueller, 1972). 
No estágio inicial, o foco da empresa é a viabilidade da sua existência e, para tal, a estratégia empresarial é identificar e captar o maior número de clientes possíveis. Nessa fase, a firma tende a possuir baixa quantidade de ativos, fazendo com que reinvistam uma alta parcela de seus lucros em investimentos de capital, ocasionando o baixo pagamento de dividendos (Aharony \& Yehuda, 2006; E. L. Black, 1998; Miller \& Friesen, 1984). Devido a problemas de restrições de caixa, a firma busca capital adicional externo, e encontrará uma demanda de exigência por maiores retornos pelos novos investidores, consequência do risco e da incerteza contidos nesse estágio (Mueller, 1972). Nesse sentido, E. L. Black (1998) enfatiza que a valuation da firma normalmente é realizada com base em suas oportunidades futuras de crescimento.

Após conseguir ultrapassar com sucesso o estágio inicial, a firma altera seu estágio para o de crescimento. Neste estágio, a empresa vê-se diante de projetos com possibilidade de valor presente líquido positivo (Grullon \& Michaely, 2004), causando crescimento rápido e vendas elevadas, aumento de contas a receber e estoques, devido a altos níveis de despesas de capital (E. L. Black, 1998) direcionados à inovação tecnológica buscando o início da diferenciação do produto (Jenkins, Kane, \& Velury, 2004). A política de financiamento, nesse estágio, é caracterizada por baixo pagamento de dividendos (E. L. Black, 1998) e os pagamentos dos gestores começam a se basear em planos de compensação por incentivos (Liao, 2008). A valuation da empresa pelos investidores é feita com base nas suas oportunidades de crescimento e da sua capacidade de gerar fluxos de caixa futuros (Aharony \& Yehuda, 2006; E. L. Black, 1998).

Após o crescimento, as firmas entram na fase de maturidade, mudando o foco de sua estratégia no crescimento das vendas para o crescimento da rentabilidade, caracterizando essa fase pela maximização da rentabilidade e da minimização dos custos (E. L. Black, 1998; Jenkins et al., 2004). Por terem feito altos investimentos em ativos no estágio anterior, há um decréscimo relativo no nível de investimento em inovações, visto que a depreciação, em si, pode ser suficiente para financiar a substituição e a manutenção de ativos, consequentemente há uma diminuição da taxa de crescimento devido à estabilização dos níveis de operações (Aharony \& Yehuda, 2006; Chiang, Lee, \& Anandarajan, 2011).

Por possuírem altos níveis de lucros acumulados, tendo menos oportunidade de investimento em relação às firmas em estágio de crescimento, as empresas maduras têm excesso de fluxo de caixa, e, para evitar conflitos de agência devido a isso, a administração opta pela recompra de ações em circulação (Aharony \& Yehuda, 2006). A empresa nesse estágio se torna mais diversificada, resultando em uma redução do custo de capital e do risco, gerando um aumento no valor da firma, como consequência. Para evitar altos pagamentos de dividendos, essas empresas procuram não reportar altos lucros, adotando um comportamento mais conservador e com gerenciamento dos resultados para baixo, com accruals negativos (Grullon \& Michaely, 2004; Shyu \& Chen, 2009; Xu, 2007).

O último estágio é o de declínio que, de acordo com Mueller (1972), faz com que as firmas passem a focar mais na minimização de custos por meio da eficiência operacional (E. L. Black, 1998; Jenkins et al., 2004). Por estar há mais tempo no mercado, existe uma pressão maior pela maximização do valor dos acionistas, e uma maior exigência de altas taxas de pagamento de dividendos (E. L. Black, 1998; Mueller, 1972; Ramaswamy, Ueng, \& Carl, 2007). Devido à estagnação e à exigência de altos dividendos, as operações menos rentáveis são excluídas da empresa e há uma diminuição no nível de dívida através de recompra de ações, como objetivo de aumentar a rentabilidade (Coulton \& Ruddock, 2011).

\section{Qualidade das informações contábeis}

A qualidade das informações contábeis tem sido um tema amplamente pesquisado, nacional e internacionalmente, nos últimos anos (Dechow et al., 2010; Dichev, Graham, Harvey, \& Rajgopal, 2013). Este interesse se deve ao fato de que a informação contábil, segundo Beyer, Cohen, Lyz e Walther (2010), desempenha dois papéis importantes no mercado de capitais: (a) auxilia na avaliação dos retornos potenciais das oportunidades de investimentos; e (b) permite ao fornecedor de capital 
(investidores ou credores) monitorar o uso dos recursos disponíveis da firma. Assim, a qualidade das informações reportadas pelas companhias é consistente com a perspectiva de valuation da firma, em que o investidor observa como ocorre a geração de lucros e fluxos de caixa ao longo da vida da firma (Dichev et al., 2013).

Não existe um consenso sobre a definição e a mensuração da qualidade das informações contábeis (earnings quality). Observa-se uma lista de medidas, tais como persistência dos lucros, gerenciamento de resultados, reconhecimento assimétrico das perdas (conservadorismo), value relevance, transparência e comparabilidade.

O conservadorismo se refere a uma característica da informação (Dichev et al., 2013) na qual se exige um maior nível de verificabilidade para os ganhos do que para as perdas (Basu, 1997; Paulo, Antunes, \& Formigoni, 2008; Watts, 2003a, 2003b). Apesar de críticas sobre o conservadorismo contábil - pois impõe o viés do reconhecimento assimétrico das perdas, mascarando o verdadeiro desempenho econômico da firma (Penman \& Zhang, 2002) - Watts (2003a) argumenta que, para fins contratuais, essa é uma característica importante, haja vista que inibe ações oportunistas por parte dos gestores.

O interesse pela persistência dos resultados contábeis é explicado pelo fato de que ela auxilia na previsão dos resultados futuros, facilitando a valuation. Segundo Dichev, Graham, Harvey e Rajgopal (2013), resultados mais persistentes tornam-se melhores preditores de lucros sustentáveis no longo prazo. Assim, erros (intencionais ou não) nas estimativas contábeis reduzem a persistência dos resultados, o que afeta negativamente a utilidade dos lucros no papel de avaliação da firma e nas previsões de lucros futuros.

O gerenciamento de resultados contábeis é a má representação intencional do desempenho econômico da firma, dentro das alternativas permitidas pelas normas contábeis, não se confundindo com fraude. $\mathrm{O}$ gerenciamento de resultados ocorre por causa da discricionariedade dos gestores no processo de elaboração dos relatórios contábeis.

A literatura corrente documenta que a qualidade das informações contábeis foi analisada com base nos vários aspectos institucionais e organizacionais, incentivos, eventos específicos, etc. (Beyer, Cohen, Lyz, \& Walther, 2010; Dechow et al., 2010; Dichev et al., 2013); mas apresenta poucas evidências sobre a sua relação com o ciclo de vida da firma.

\section{Evidências empíricas}

Segundo Drake (2013), "a teoria do ciclo de vida prediz que os participantes do mercado têm expectativas diferentes e reagem diferentemente sobre o desempenho da firma ao longo das fases do ciclo de vida" (p. 27), avaliando lucros, vendas, fluxos de caixa e accruals de forma distinta em cada estágio.

Algumas pesquisas (Anthony \& Ramesh, 1992; E. L. Black, 1998; Jenkins et al., 2004) investigaram a relação entre retorno de ações e qualidade dos lucros em diferentes estágios de ciclo de vida das empresas, e os resultados evidenciaram que, nesses diferentes estágios, as firmas apresentaram características econômicas e financeiras distintas, ao passo que aquelas em estágios semelhantes compartilharam características similares.

No que se refere aos relatórios contábeis durante o período de crescimento, pode-se identificar a existência de menos persistência no lucro (E. L. Black, 1998) e grande quantidade de accruals positivos (Liao, 2008; Liu, 2008); consequentemente, identifica-se uma contabilidade menos conservadora (Park \& Chen, 2006), pois os accruals positivos fazem com que o lucro seja aumentado.

$\mathrm{Na}$ fase de maturidade, para evitar altos pagamentos de dividendos, as empresas evitam reportar altos lucros, adotando um comportamento mais conservador e com gerenciamento dos resultados para baixo (Grullon \& Michaely, 2004; Shyu \& Chen, 2009; Xu, 2007). Dickinson (2011) evidenciou que 
existe maior persistência nos lucros antes dos tributos na fase de maturidade. Já na fase de declínio, Liu (2008) observou que as características das empresas nesse estágio têm uma maior propensão à apresentação de accruals negativos.

Como as empresas em fase de crescimento precisam obter mais recursos para financiar novos projetos, elas buscam divulgar maiores lucros com o intuito de atrair mais investidores; assim, os gestores têm maiores incentivos para reportar informações contábeis menos conservadoras. Contudo, existem evidências de que empresas mais conservadoras tendem a criar mais valor no longo prazo (Feltham \& Ohlson, 1995; Penman \& Zhang, 2002), sendo que um peso maior é dado pelos investidores para os lucros operacionais anormais de empresas mais conservadoras do que as menos conservadoras (Ahmed et al., 2000; Stober, 1996). Desse modo, têm-se incentivos para a utilização de práticas conservadoras na mensuração dos lucros para as companhias em estágio de crescimento.

Alguns trabalhos têm confirmado que empresas em estágios mais avançados do ciclo de vida tendem a ser mais conservadoras do que aquelas que se encontram em estágios iniciais. Miller e Friesen (1984) afirmam que as empresas em maturidade e em declínio são mais conservadoras, focando na maximização do reconhecimento de perdas e despesas, enquanto as companhias em crescimento focam na maximização do reconhecimento de receitas.

Apesar de ser esperado, teoricamente, que as empresas em estágio de crescimento tenham um comportamento menos conservador, para aumentar seus lucros, existem evidências de que elas se comportam de forma mais conservadora (Ball \& Shivakumar, 2008), e têm incentivos para isso, como citado anteriormente (Feltham \& Ohlson, 1995; Penman \& Zhang, 2002). Assim, espera-se que as empresas em estágio de crescimento sejam mais conservadoras quanto à mensuração dos seus ativos operacionais do que as empresas em estágio de declínio, de modo a maximizar o seu lucro operacional anormal futuro. Porém, quando da mensuração do lucro líquido, era esperado que as empresas em estágio de declínio fossem mais conservadoras, para evitar o pagamento de dividendos e tributos.

Liu (2008) argumenta que os accruals discricionários com viés positivo nas empresas que participaram de emissão inicial de ações (IPO) e com viés negativo das empresas que estão apresentando ativos em desvalorização não estão relacionados aos eventos em si, IPO ou ativos se desvalorizando, mas em relação ao estágio do ciclo de vida das empresas (fase de crescimento ou fase de declínio), corroborando Ball e Shivakumar (2008) sobre o viés positivo dos accruals discricionários das empresas em IPO não ser necessariamente relacionado ao gerenciamento de resultados, bem como está em acordo com Dechow e Ge (2006) sobre o viés negativo das empresas em declínio. Além disso, Ball e Shivakumar (2008) enfatizam que os accruals dependem do ciclo de vida das empresas.

Algumas evidências são apontadas, por exemplo, nas pesquisas sobre gerenciamento de resultados nos IPOs, em que as companhias que emitem novos títulos possuem um maior nível de discricionariedade em seus resultados (Teoh, Welch, \& Wong, 1998; Teoh, Wong, \& Rao, 1998). Por outro lado, no caso brasileiro, existem evidências de que não há variação no gerenciamento dos resultados, nem na qualidade dos accruals em torno do IPO (Paulo, Cavalcante, \& Melo, 2012).

\section{Procedimentos Metodológicos}

\section{População e amostra}

O presente estudo utilizou dados das demonstrações contábeis disponíveis na Economatica ${ }^{\circledR}$ e nos formulários de referência de 605 empresas listadas na BM\&FBOVESPA, classificadas em 19 setores, durante o período de 1995 a 2011.

Foram excluídas da amostra as empresas do setor financeiro, porque seus processos de mensuração de accruals se diferenciam substancialmente das demais companhias e, provavelmente, não 
são capturados adequadamente pelos modelos analisados (Peasnell, Pope, \& Young, 2000), bem como o seu elevado grau de alavancagem financeira, que poderia ser interpretado como dificuldades financeiras junto às demais empresas. Além disso, as empresas com ativos operacionais líquidos negativos também foram excluídas, pois a inclusão delas afetaria o cálculo do lucro operacional anormal pelo modelo de Feltham e Ohlson (1995). Em média, foram incluídas 170 empresas por ano.

\section{Definição dos estágios de ciclo de vida}

Devido à ocorrência de muitas fusões no mercado de capitais brasileiro, e considerando que o número de companhias abertas no Brasil é significativamente menor em comparação aos ambientes analisados pelas pesquisas internacionais, optou-se pela não exclusão das firmas na fase inicial. Além disso, E. L. Black (1998) pressupõe que a empresa não entra no estágio de crescimento com menos de três anos desde a sua fundação. Assim, nesta pesquisa, todas as observações foram classificadas nos estágios: crescimento (que inclui o estágio inicial), maturidade e declínio.

Para identificar o estágio de ciclo de vida de cada empresa, adotou-se a metodologia utilizada por Anthony e Ramesh (1992), E. L. Black (1998), Park e Chen (2006) e demais pesquisas apresentadas anteriormente para justificar as variáveis escolhidas (Aharony \& Yehuda, 2006; Coulton \& Ruddock, 2011; Grullon \& Michaely, 2004; Jenkins et al., 2004; Miller \& Friesen, 1984; Mueller, 1972; Ramaswamy et al., 2007).

Inicialmente, as empresas foram divididas por setores e, em seguida, foram identificados os quintis das proxies utilizadas para classificar os estágios do ciclo de vida da empresa, utilizando o cálculo dos índices da Tabela 1: (a) despesas de capital (CEV); (b) crescimento das vendas (SG); (c) payout dos dividendos (DP); e (d) idade da empresa (AGE). Em cada ano, as companhias foram classificadas em: (a) crescimento; (b) maturidade; ou (c) declínio. Esse procedimento busca a segurança de que múltiplas variáveis possam eliminar ou minimizar o viés de se ter apenas uma variável indicativa do estágio de ciclo de vida.

Tabela 1

Variáveis Utilizadas para a Classificação dos Estágios de Ciclo de Vida

\begin{tabular}{lc}
\hline Despesas de Capital & $C E V=\frac{\text { Capex }_{t}}{\text { PatrimônioLiquido } \text {. } 100}$ \\
Crescimento das Vendas & $S G=\frac{\text { VendasLíquidas }_{t}-\text { VendasLíquidas }_{t-1}}{\text { VendasLíquidas }_{t-1}}$ \\
Payout dos dividendos & $D P=\frac{\text { DividendosPagos }_{t}}{\text { LucroLíquido } 100}$ \\
Idade da empresa & $A G E=$ Ano atual - Ano da fundação \\
\hline
\end{tabular}

Nota. Fonte: Adaptado de Park, Y., \& Chen, K. H. (2006). The effect of accounting conservatism and life-cycle stages on firm valuation (p. 81). Journal of Applied Business Research, 22(3), 75-92.

Resumidamente, aplicou-se a seguinte metodologia de classificação: a empresa foi classificada no estágio de crescimento quando localizada no quintil mais alto ( $5^{\circ}$ quintil), no que se refere ao crescimento das vendas e ao custo de capital, e no quintil mais baixo em relação ao pagamento de dividendos e à idade da empresa ( $1^{\circ}$ quintil). O total do somatório das maiores pontuações será igual a 20 pontos. A empresa foi classificada no estágio de maturidade quando o crescimento das vendas e do custo de capital se apresentou situado no $3^{\circ}$ quintil, bem como no $4^{\circ}$ quintil a idade e, no $5^{\circ}$ quintil, no que se refere ao pagamento de dividendos. $\mathrm{O}$ total do somatório das maiores pontuações nesse estágio será de 15 pontos. A empresa foi classificada no estágio de declínio quando situada no quintil com a pontuação mais baixa ( $1^{\circ}$ quintil) no que se refere ao crescimento de vendas e à despesa de capital. Já em relação ao pagamento dos dividendos e à idade, a empresa foi classificada no $2^{\circ}$ e no $5^{\circ}$ quintil, 
respectivamente. $\mathrm{O}$ total do somatório das maiores pontuações nesse estágio será de 8 pontos. A Tabela 2 apresenta um resumo desta metodologia.

Tabela 2

Classificação dos Estágios de Ciclo de Vida

\begin{tabular}{lccccc}
\hline Quintis & \multicolumn{4}{c}{ Descrição dos Ciclos de Vida } & \\
& & DP & SG & CEV & AGE \\
\hline $1^{\circ}$ quintil & $0 \%-20 \%$ & $5(1)$ & 1 & 1 & 5 \\
$2^{\circ}$ quintil & $20 \%-40 \%$ & $4(2)$ & 2 & 2 & 4 \\
$3^{\circ}$ quintil & $40 \%-60 \%$ & 3 & 3 & 3 & 3 \\
$4^{\circ}$ quintil & $60 \%-80 \%$ & 3 & 4 & 4 & 2 \\
$5^{\circ}$ quintil & $80 \%-100 \%$ & 3 & 5 & 5 & 1 \\
\hline
\end{tabular}

Nota. Fonte: Adaptado de Park, Y., \& Chen, K. H. (2006). The effect of accounting conservatism and life-cycle stages on firm valuation (p. 82). Journal of Applied Business Research, 22(3), 75-92.

Após o somatório dos quintis de cada empresa em cada ano, estas foram classificadas conforme Park e Chen (2006), possibilitando a identificação do estágio do ciclo de vida. Quando o somatório dos fatores estiver entre dezesseis e vinte, a empresa é classificada no estágio de crescimento. Se o somatório estiver entre nove e quinze, é considerado que a empresa está no estágio de maturidade; e se a soma for entre quatro e oito, classifica-se a empresa no estágio de declínio.

\section{Definição das hipóteses de pesquisa e modelos operacionais empregados}

Baseado em diversas pesquisas sobre ciclo de vida (E. L. Black, 1998; Jenkins et al., 2004; Ou \& Penman, 1989; Park \& Chen, 2006; Penman \& Zhang, 2002; Xu, 2007), foi identificado o comportamento esperado dos atributos da qualidade das informações contábeis em cada um dos estágios do ciclo de vida na empresa (Figura 1).

\begin{tabular}{|c|c|c|c|c|}
\hline $\begin{array}{c}\text { Estágio do } \\
\text { Ciclo de Vida }\end{array}$ & Conservadorismo & $\begin{array}{c}\text { Persistência } \\
\text { dos-Lucros } \\
\text { Operacionais }\end{array}$ & $\begin{array}{c}\text { Persistência } \\
\text { dos Fluxos de } \\
\text { Caixa }\end{array}$ & $\begin{array}{c}\text { Gerenciamento } \\
\text { dos } \\
\text { Resultados }\end{array}$ \\
\hline Crescimento & - & + & + & + \\
\hline
\end{tabular}

Figura 1. Relação da Qualidade das Informações Contábeis com os Estágios do Ciclo de Vida

\section{Conservadorismo}

Como descrito anteriormente no referencial teórico, os incentivos distintos dos gestores em cada um dos estágios do ciclo de vida afetam diferentemente o nível de conservadorismo dos números contábeis (Ahmed et al., 2000; Ball \& Shivakumar, 2008; Feltham \& Ohlson, 1995; Grullon \& Michaely, 2004; Lev, Sarath, \& Sougiannis, 2005; Miller \& Friesen, 1984; Park \& Chen, 2006; Penman \& Zhang, 2002; Shyu \& Chen, 2009; Stober, 1996; Xu, 2007). Assim, tem-se a seguinte hipótese de pesquisa: 
Hipótese 1: Existem diferenças significativas entre o conservadorismo contábil das companhias nos estágios de crescimento, maturidade e declínio.

Para capturar o comportamento conservador das firmas nos diferentes estágios do ciclo de vida, utilizaram-se os modelos de Basu (1997) e Feltham e Ohlson (1995), doravante MFO. O modelo proposto por Basu (1997) é um dos mais utilizados nas pesquisas empíricas nacionais e internacionais, sendo descrito da seguinte forma (equação 1):

$$
\frac{L L_{i t}}{P_{i t-1}}=\alpha_{0}+\alpha_{1} D R_{i t}+\alpha_{2} R_{i t}+\alpha_{3} R_{i t}^{*} D R_{i t}+\varepsilon_{i t}
$$

Em que: $L L_{i t}$ é o lucro líquido contábil por ação da empresa $i$ no ano $t ; P_{i t-1}$ é o preço da ação da empresa $i$ no ano $t-1 ; D R_{i t}$ é uma dummy para indicar se o retorno foi negativo, assumindo valor 1 se $R_{i t}$ $<0$ e 0 nos demais casos; $R_{i t}$ é o retorno da ação da empresa $i$ no ano $t ; \varepsilon_{i t}$ é o erro da regressão.

O coeficiente $\alpha_{3}$ do modelo de Basu (1997) é a proxy para o conservadorismo contábil e mensura a intensidade da assimetria no reconhecimento de boas e más notícias, de modo que valores maiores e mais significativos para $\alpha_{3}$ indicam que as companhias incluídas na amostra têm, em média, um alto grau de conservadorismo $\left(\alpha_{3}>0\right)$. Para efeito de teste das hipóteses, considera-se que a amostra (empresas em crescimento, maduras ou em declínio) com o maior $\alpha_{3}$ será a mais conservadora.

O segundo modelo utilizado para medir o conservadorismo foi o desenvolvido por Feltham e Ohlson (1995). O MFO é um modelo linear que relaciona um conjunto de informações, as quais contemplam o valor contábil e o lucro residual para atividades operacionais. A importância fundamental do MFO é que ele incorpora o conservadorismo contábil no processo de avaliação patrimonial, possibilitando a sua utilização para a medição do conservadorismo contábil sob a perspectiva interna (em relação aos lucros operacionais anormais) e externa (em relação ao valor de mercado).

Dessa forma, quanto à perspectiva interna e externa, respectivamente, esta pesquisa utilizou as equações 2 e 3, baseando-se nas dinâmicas das informações lineares e na equação de avaliação da empresa no MFO, para medir o conservadorismo contábil.

$$
\begin{aligned}
& L O A_{i t+1}=\alpha_{0}+\alpha_{1} L O A_{i t}+\alpha_{2} A O L_{i t}+\varepsilon_{i t} \\
& V M_{i t}=\alpha_{0}+\alpha_{3} L O A_{i t}+\alpha_{4} A O L_{i t}{ }^{+} \varepsilon_{i t}
\end{aligned}
$$

Em que $L O A_{i t}$ representa o lucro operacional anormal da empresa $i$ no ano $t$. $A O L_{i t}$ são os ativos operacionais líquidos da empresa $i$ no ano $t ; V M_{i t}$ é o valor de mercado da empresa $i$ coletado em $1^{\circ}$ de abril (tolerância de 15 dias) do ano $t+1$; é $\varepsilon_{i t} \mathrm{O}$ erro da regressão. Todas as variáveis foram calculadas conforme Feltham e Ohlson (1995) e foram ponderadas pelos ativos totais das companhias no início do ano em análise.

O LOA é calculado pela diferença entre o lucro operacional corrente e os ativos operacionais líquidos do início do período multiplicados por uma taxa em nível de custo do capital. Em 7 dos 17 anos analisados foram encontrados retornos anuais negativos no principal bechmark brasileiro (Ibovespa); por este motivo, optou-se por utilizar a taxa Selic (taxa overnight do Sistema Especial de Liquidação e de Custódia) de cada ano como custo do capital (Fraletti, 2004).

As empresas que reportam seus lucros de forma mais conservadora tendem a subestimar os seus ativos operacionais atuais (Park \& Chen, 2006), o que faz com que elas tenham um maior potencial para gerar lucros operacionais anormais (LOA) futuros, considerando os ativos operacionais divulgados contemporaneamente nas demonstrações contábeis, visto que o LOA é obtido por meio da diferença entre lucro operacional e o ativo operacional do início do período multiplicado por uma taxa que represente o custo do capital da firma. 
Assim, se o parâmetro de conservadorismo da equação 2 for igual a zero $\left(\alpha_{2}=0\right)$, o tratamento contábil é considerado sem viés, sendo que, se for menor que zero $\left(\alpha_{2}<0\right)$, o tratamento contábil dos lucros é considerado agressivo; caso seja maior que zero $\left(\alpha_{2}>0\right)$, o tratamento contábil é considerado conservador, ou seja, os ativos operacionais estão sendo subestimados, considerando a explicação dos LOA futuros. A mesma ideia é utilizada na perspectiva do mercado, na equação 3. Se o coeficiente $\alpha_{4}$ for maior que 1 , implica dizer que o mercado percebe que o ativo deveria ser mensurado a um montante maior do que foi reportado pela firma, logo, a mensuração foi feita de forma conservadora $\left(\alpha_{4}>1\right)$.

Esses modelos utilizados divergem do MFO original pelo fato de que, no presente estudo, não foram utilizadas as variáveis que refletem outras informações, de modo que o termo de erro da regressão poderá ser majorado.

\section{Persistência dos lucros e fluxos de caixa}

Considerando que a persistência dos resultados contábeis é importante para o processo de valuation e para a avaliação de desempenho, que os lucros são mais persistentes do que os fluxos de caixas (Dechow et al., 2010; Dechow \& Schrand, 2004), que algumas pesquisas sugerem que empresas em fase de declínio (pela redução de valor dos seus ativos) apresentam desempenho fraco em relação aos lucros (Riedl, 2004), bem como as demais pesquisas que tratam da relação entre os estágios do ciclo de vida e a persistência dos lucros (E. L. Black, 1998; Dickinson, 2011; Drake, 2013), têm-se as seguintes hipóteses:

Hipótese 2a: Os resultados contábeis reportados pelas firmas apresentam maior persistência do que os fluxos de caixa.

Hipótese 2b: O comportamento dos resultados contábeis é mais persistente nas empresas em fase de crescimento do que nas empresas em fase de declínio.

A persistência dos resultados e dos fluxos de caixa foi medida pelo modelo padrão de estimativa da persistência, conforme a equação 4 (Dechow \& Schrand, 2004), sendo que quanto maior for o $\alpha_{1}$, mais persistente é o lucro operacional ou o fluxo de caixa:

$$
X_{i t+1}=\alpha_{0}+\alpha_{1} X_{i t}+\varepsilon_{i t}
$$

Em que: $X_{i t+l}$ é o valor da variável (lucro ou fluxo de caixa operacional) na empresa $i$ do ano $t+1$; $X_{i t}$ é o valor da variável (lucro ou fluxo de caixa operacional) na empresa $i$ do ano $t$; e $\varepsilon_{i t}$ é o erro da regressão. Todas as variáveis são ponderadas pelos ativos totais no início do período.

As empresas que estão nos estágios de crescimento (por incorrem em grandes despesas de capital, e.g. para explorar oportunidades de lucros futuros, reportando maiores perdas e prejuízos correntes) e declínio (por não terem oportunidades de crescimento para explorar) tendem a ter perdas reportadas com maior frequência do que as empresas que estão no estágio de maturidade (E. L. Black, 1998). Contudo, como a persistência dos lucros é uma qualidade necessária para os inputs de alguns modelos de avaliação de empresas, e as companhias em fase de crescimento precisam de investidores que avaliarão a empresa antes de aplicarem seus recursos, é provável que seus lucros sejam persistentes. Dessa forma, espera-se que os lucros das empresas em fase de crescimento sejam mais persistentes do que os das empresas em estágio de declínio.

\section{Gerenciamento de resultados contábeis}

De acordo com as pesquisas apresentadas anteriormente (Ball \& Shivakumar, 2008; Dechow \& Ge, 2006; Grullon \& Michaely, 2004; Shyu \& Chen, 2009; Xu, 2007), o comportamento dos accruals discricionários pode estar relacionado com os estágios do ciclo de vida das firmas. Diante do exposto, tem-se a seguinte hipótese de pesquisa: 
Hipótese 3: O comportamento do gerenciamento de resultados varia significativamente ao longo do ciclo de vida das empresas.

Para analisar a hipótese acima, foi utilizado o modelo de Pae (2005), descrito na equação 5, por ter sido o primeiro a considerar a reversão dos accruals de um período para o outro:

$$
T A_{i t}=\alpha\left(\frac{1}{A_{t-1}}\right)+\beta_{1}\left(\Delta R_{i t}\right)+\beta_{2}\left(P P E_{i t}\right)+\beta_{3}\left(F C O_{i t}\right)+\beta_{4}\left(F C O_{i t-1}\right)+\beta_{5}\left(T A_{i t-1}\right)+\varepsilon_{i t}
$$

Em que: $T A_{i t}$ são os accruals totais da empresa $i$ no ano $t ; \Delta R_{i t}$ a variação das receitas líquidas da empresa $i$ do período $t-1$ para o período $t$; $P P E_{i t}$ são os saldos das contas Ativo Imobilizado da empresa $i$ no final do período $t ; A_{i t-1}$ são os ativos totais da empresa no final do período $t-1 ; F C O_{i t}$ é fluxo de caixa operacional da empresa $i$ no período $t ; F C O_{i t-1}$ é fluxo de caixa operacional da empresa $i$ no período $t$-1; $T A_{i t-1}$ accruals totais da empresa $i$ no período $t$; e $\varepsilon_{i t}$ é o erro da regressão. Todas as variáveis são ponderadas pelos ativos totais no início do período.

Os accruals totais são calculados da seguinte forma:

$$
T A_{i t}=\left(\Delta A C_{i t}-\Delta D i s p_{i t}\right)-\left(\Delta P C_{i t}-\Delta D i v_{i t}\right)-D e p r_{i t}
$$

Em que: $T A_{i t}$ são os accruals totais da empresa no período $t ; \Delta A C_{i t}$ é a variação do ativo corrente da empresa $i$ no final do período $t$-1 para o final do período $t ; \Delta P C_{i t}$ é a variação do passivo corrente da empresa $i$ no final do período $t-1$ para o final do período $t ; \Delta D i s p_{i t}$ é variação das disponibilidades da empresa $i$ no final do período t-1 para o final do período t; $\Delta D i v_{i t}$ é a variação dos financiamentos e empréstimos de curto prazo da empresa $i$ no final do período $t-1$ para o final do período $t$; Depr $i t$ é o montante das despesas com depreciação e amortização da empresa $i$ durante o período $t$. Todas as variáveis são ponderadas pelos ativos totais no início do período $t$.

$\mathrm{Na}$ análise das possíveis diferenças de gerenciamento de resultados entre os diferentes estágios de ciclo de vida, considerou-se que a amostra com o maior desvio-padrão nos accruals discricionários (termo de erro da equação 5) é o estágio do ciclo de vida com a maior probabilidade das empresas estarem gerenciando seus resultados contábeis. Além disso, analisou-se a média e a mediana dos accruals discricionários para avaliar qual estágio se gerencia o resultado, para mais ou para menos, esperando que as empresas em crescimento gerenciem para mais (accruals discricionários positivos) e as que estão em declínio gerenciem para menos (accruals discricionários negativos), de modo a atrair mais investidores com maiores lucros e pagar menos dividendos, respectivamente.

Para analisar se o comportamento do gerenciamento de resultados é significativamente diferente entre os estágios do ciclo de vida das empresas contidas na amostra, foi utilizado o teste Chi-quadrado de diferença de média, Kruskal-Wallis, uma vez que a hipótese de normalidade e heterogeneidade dos accruals foi rejeitada no nível de 5\% pelos testes de Kolmogorov-Smirnov e de Levene.

Além disso, para controlar possíveis efeitos da endogeneidade das variáveis e conferir maior robustez às estimações, foi utilizado adicionalmente o Método dos Momentos Generalizados (MMG). Nos casos onde o MMG foi necessário, adicionou-se uma nota de tabela, apontando o efeito do método de estimação nos resultados. 


\section{Apresentação e Análise dos Resultados}

\section{Conservadorismo}

As Tabelas 3 e 4 apresentam os resultados relacionados ao conservadorismo contábil na mensuração dos ativos operacionais na perspectiva interna e na perspectiva do mercado por meio do MFO.

Tabela 3

Conservadorismo na Perspectiva Interna pelo Modelo de Feltham e Ohlson (1995)

\begin{tabular}{lcccccccc}
\hline LOA $_{\text {it+1 }}$ & \multicolumn{1}{c}{ Completa $^{\mathbf{b}, \mathbf{c}}$} & \multicolumn{3}{c}{ Crescimento $^{\mathbf{c}}$} & \multicolumn{3}{c}{ Maturidade $^{\mathbf{b}, \mathbf{c}}$} & \multicolumn{3}{c}{ Declínio $^{\mathbf{b}, \mathbf{c}}$} \\
\cline { 2 - 8 } & coefic. & p-valor & coefic. & p-valor & coefic. & p-valor & coefic. & p-valor \\
\hline Constante & 0,033 & 0,000 & 0,056 & 0,000 & 0,038 & 0,000 & 0,027 & 0,002 \\
LOA $_{\text {it }}$ & 0,575 & 0,000 & 0,423 & 0,000 & 0,589 & 0,000 & 0,516 & 0,000 \\
AOL $_{i t}$ & $-0,049$ & 0,000 & $-0,062$ & 0,015 & $-0,044$ & 0,000 & $-0,071$ & 0,000 \\
R $^{2}$ ajustado & 0,419 & - & 0,405 & - & 0,426 & - & 0,366 & - \\
Teste F & 680,269 & 0,000 & 29,877 & 0,000 & 441,895 & 0,000 & 141,657 & 0,000 \\
Teste White & 31,262 & 0,000 & 16,489 & 0,005 & 29,550 & 0,000 & 11,221 & 0,047 \\
Teste BG & 69,781 & 0,000 & 5,213 & 0,022 & 38,852 & 0,000 & 15,520 & 0,000 \\
Teste JB & 75,401 & 0,000 & 0,366 & 0,832 & 35,222 & 0,000 & 35,049 & 0,000 \\
N & 1.883 & & 86 & & 1.189 & & 487 & \\
\hline
\end{tabular}

Nota. O grau de multicolinearidade, pelo fator de inflação da variância, não é considerado problemático no modelo: 1,020 (pooled), 1,034 (crescimento), 1,018 (maturidade) e 1,007 (declínio).

${ }^{a}$ Corrigido por White; ${ }^{b}$ De acordo com o teorema do limite central, o pressuposto da normalidade foi relaxado; ${ }^{\mathrm{c}}$ Corrigido por Newey-West.

Tabela 4

Conservadorismo na Perspectiva Externa pelo Modelo de Feltham e Ohlson (1995)

\begin{tabular}{|c|c|c|c|c|c|c|c|c|}
\hline \multirow[t]{2}{*}{$\mathbf{V M}_{\mathrm{it}}$} & \multicolumn{2}{|c|}{ Completa $^{\mathrm{b}, \mathrm{c}}$} & \multicolumn{2}{|c|}{ Crescimento $^{\mathrm{b}, \mathrm{c}}$} & \multicolumn{2}{|c|}{ Maturidade $^{b, c}$} & \multicolumn{2}{|c|}{ Declínio $^{\text {b, c }}$} \\
\hline & coefic. & p-valor & coefic. & p-valor & coefic. & p-valor & coefic. & p-valor \\
\hline Constante & 0,376 & 0,000 & 0,233 & 0,141 & 0,412 & 0,000 & 0,330 & 0,000 \\
\hline $\mathrm{LOA}_{\text {it }}$ & 2,790 & 0,000 & 4,120 & 0,000 & 3,426 & 0,000 & 2,141 & 0,000 \\
\hline $\mathrm{AOL}_{\text {it }}$ & 0,491 & 0,000 & 0,539 & 0,053 & 0,438 & 0,000 & 0,526 & 0,000 \\
\hline $\mathrm{R}^{2}$ ajustado & 0,203 & - & 0,285 & - & 0,228 & - & 0,218 & - \\
\hline Teste F & 242,631 & 0,000 & 18,220 & 0,000 & 177,242 & 0,000 & 69,975 & 0,000 \\
\hline Teste White & 58,416 & 0,000 & 18,545 & 0,002 & 58,497 & 0,000 & 21,463 & 0,000 \\
\hline Teste BG & 73,182 & 0,000 & 48,819 & 0,000 & 58,855 & 0,000 & 14,253 & 0,000 \\
\hline Teste JB & 1727,923 & 0,000 & 23,586 & 0,000 & 1199,384 & 0,000 & 242,320 & 0,000 \\
\hline $\mathrm{N}$ & 1.896 & & 97 & & 1.192 & & 494 & \\
\hline
\end{tabular}

Nota. O grau de multicolinearidade, pelo fator de inflação da variância, não é considerado problemático no modelo: 1,017 (pooled), 1,020 (crescimento), 1,015 (maturidade) e 1,055 (declínio).

${ }^{\text {a }}$ Corrigido por White; ${ }^{\mathrm{b}}$ De acordo com o teorema do limite central, o pressuposto da normalidade foi relaxado; ${ }^{\mathrm{c}}$ Corrigido por Newey-West. 
Analisando os resultados apresentados na Tabela 3, observa-se que as empresas listadas na amostra completa têm seus ativos operacionais mensurados de forma agressiva, na perspectiva interna, uma vez que o coeficiente da variável AOL foi significativamente menor que zero. Esse resultado se confirma quando se analisam as amostras das firmas classificadas entre os estágios do ciclo de vida. A distância para menos da variável AOL, em relação a zero, indica que a mensuração dos ativos operacionais líquidos é efetuada de forma mais agressiva, enquanto que a distância para mais indica que a mensuração foi mais conservadora, o que aponta para um maior conservadorismo das empresas em estágio de crescimento, em relação às que estão no estágio de declínio.

Na perspectiva do mercado (Tabela 3), a mensuração dos ativos operacionais líquidos foi considerada agressiva, uma vez que o coeficiente da variável AOL foi significativamente menor que 1. Contudo, pode-se observar que, ao nível de significância de 10\%, as empresas listadas no estágio de crescimento são mais conservadoras que as demais, visto que o seu coeficiente foi mais próximo a 1 do que as firmas dos demais estágios.

Os resultados apresentados nas Tabelas 3 e 4 estão dentro do que era esperado com base no referencial teórico, visto que as empresas em crescimento buscam maximizar seus lucros operacionais anormais futuros (Ahmed et al., 2000; Stober, 1996), precisando então mensurar seus ativos operacionais líquidos de forma conservadora, implicando em criação de valor no longo prazo (Feltham \& Ohlson, 1995; Penman \& Zhang, 2002).

Apesar de os resultados apresentados nas Tabelas 3 e 4 apontarem evidências de que as empresas em estágio de crescimento mensuram os seus ativos operacionais líquidos de forma mais conservadora do que as empresas em estágio de declínio, a análise feita por meio do modelo de Basu (1997) na Tabela 5 aponta que as empresas em declínio têm seus lucros mensurados de forma mais conservadora do que as demais, enquanto que as empresas em estágio de crescimento e maturidade mensuram seus lucros de forma não viesada (coeficientes sem significância estatística). Esperava-se que as empresas em estágio de crescimento fossem também conservadoras quanto à mensuração do lucro líquido, de modo a evitar o pagamento de dividendos e tributos sobre o lucro no curto prazo (Ball \& Shivakumar, 2008).

Tabela 5

Conservadorismo Contábil por Meio do Modelo de Basu (1997)

\begin{tabular}{lcccccccc} 
& \multicolumn{1}{c}{ Completa $^{\mathbf{b}}$} & \multicolumn{3}{c}{ Crescimento } & \multicolumn{3}{c}{ Maturidade $^{\mathbf{a}, \mathbf{b}}$} & \multicolumn{3}{c}{ Declínio $^{\mathbf{b}}$} \\
\cline { 2 - 9 } & coefic. & p-valor & coefic. & p-valor & coefic. & p-valor & coefic. & p-valor \\
\hline Constante & 0,030 & 0,030 & 0,153 & 0,000 & 0,534 & 0,184 & $-0,109$ & 0,109 \\
DR $_{\text {it }}$ & 0,016 & 0,529 & $-0,067$ & 0,120 & $-0,643$ & 0,145 & 0,116 & 0,341 \\
$\mathrm{R}_{\text {it }}$ & 0,044 & 0,005 & 0,035 & 0,129 & $-0,821$ & 0,153 & $-0,047$ & 0,574 \\
$\mathrm{R}_{\text {it }}{ }^{*} \mathrm{DR}_{\text {it }}$ & 0,325 & 0,000 & $-0,068$ & 0,569 & 0,867 & 0,279 & 1,096 & 0,000 \\
$\mathrm{R}^{2}$ ajustado & 0,043 & - & 0,113 & - & 0,025 & - & 0,030 & - \\
Teste F & 40,007 & 0,000 & 4,687 & 0,004 & 15,209 & 0,000 & 8,136 & 0,000 \\
Teste White & 9,835 & 0,080 & 8,437 & 0,133 & 40,172 & 0,000 & 2,377 & 0,794 \\
Teste BG & 0,110 & 0,740 & 0,546 & 0,459 & 0,008 & 0,928 & 2,482 & 0,115 \\
Teste JB & 51394,85 & 0,000 & 4,829 & 0,089 & 9819,808 & 0,000 & 10340,26 & 0,000 \\
$\mathrm{~N}$ & 2.592 & & 87 & & 1.641 & & 690 & \\
\hline
\end{tabular}

Nota. O grau de multicolinearidade, pelo fator de inflação da variância, não é considerado problemático no modelo: 2,896 , 2,096 e 2,622 (pooled), 3,260, 1,845 e 2,971 (crescimento), 2,563, 1,414 e 2,410 (maturidade) e 2,876, 2,234 e 2,672 (declínio). $\mathrm{Na}$ amostra "Crescimento", a variável $\mathrm{DR}_{\text {it }}$ foi significativa ao nível de 10\% (p-valor =0,090), pelo MMG.

${ }^{\mathrm{a}}$ Corrigido por White; ${ }^{\mathrm{b}}$ De acordo com o teorema do limite central, o pressuposto da normalidade foi relaxado. 
Essas evidências podem sugerir que, apesar das oportunidades de projetos com VPL positivo, as empresas em crescimento não apresentaram comportamento conservador na mensuração do lucro líquido de forma a reduzir o pagamento de dividendos e/ou tributos. Isso pode ainda ter ocorrido pelo fato de que o lucro é uma medida utilizada estimar diversos indicadores de desempenho e está relacionada com o preço das ações (Ohlson, 1995), evidência confirmada e.g. por Lopes, Sant'Anna e Costa (2007) no Brasil.

É importante ressaltar e ter cuidado ao analisar esses resultados em conjunto visto que os modelos usam medidas diferentes para mensurar o conservadorismo - uma com base nos resultados operacionais anormais e nos ativos operacionais líquidos e outra com base no lucro líquido e no retorno das ações das empresas. Como o modelo de Basu (1997) é baseado no lucro líquido, esse resultado mais conservador pode indicar que as empresas em estágio de declínio têm um reporte do lucro líquido mais conservador para evitar o pagamento de dividendos e assim se manter por mais tempo em continuidade. Essa suposição é suportada pelo fato de que as empresas em estágio de declínio têm, em média, menores lucros (em 75\%) e menores fluxos de caixa (em 38\%) do que as empresas na fase de crescimento (com base nos dados brutos desta pesquisa, não tabulados aqui).

Assim não se pode rejeitar a hipótese 1 desta pesquisa, uma vez que há diferenças no conservadorismo entre os estágios dos ciclos de vida, e se pode inferir que, no caso dos ativos operacionais líquidos, as empresas em crescimento são mais conservadoras do que as empresas em declínio, como era esperado, uma vez que elas têm incentivos para tal prática (Feltham \& Ohlson, 1995; Penman \& Zhang, 2002).

\section{Persistência dos lucros e dos fluxos de caixa}

Analisando a persistência dos lucros individualmente e em comparação com os fluxos de caixa, testaram-se a hipótese $2 \mathrm{a}$ e $2 \mathrm{~b}$, e as estimativas dos modelos utilizados estão descritas nas Tabelas 6 e 7. Por meio dos resultados apresentados na Tabela 6 , observa-se que a persistência dos lucros é diferente entre os estágios de ciclo de vida das empresas e que os lucros são mais persistentes nas empresas em estágio de maturidade, corroborando evidências empíricas anteriores (Dechow \& Schrand, 2004; Dickinson, 2011), uma vez que a empresa começa a se posicionar melhor no mercado e a ter projetos mais consistentes, assumindo menos risco e, consequentemente, menor volatilidade nos resultados.

Tabela 6

Persistência dos Lucros Operacionais

\begin{tabular}{|c|c|c|c|c|c|c|c|c|}
\hline & Completa $^{b}$ & & Crescimento & & Maturidade $^{\mathrm{a}, \mathrm{b}}$ & & Declínio $^{b}$ & \\
\hline & coefic. & $\mathrm{p}$-valor & coefic. & $\mathrm{p}$-valor & coefic. & p-valor & coefic. & $\mathrm{p}$-valor \\
\hline Constante & 0,022 & 0,000 & 0,053 & 0,000 & 0,034 & 0,000 & 0,008 & 0,000 \\
\hline $\mathrm{LO}_{\text {it }}$ & 0,623 & 0,000 & 0,596 & 0,000 & 0,605 & 0,000 & 0,585 & 0,000 \\
\hline $\mathrm{R}^{2}$ ajustado & 0,391 & - & 0,354 & - & 0,366 & - & 0,382 & - \\
\hline Teste F & $2.939,181$ & 0,000 & 96,380 & 0,000 & $1.509,336$ & 0,000 & 700,223 & 0,000 \\
\hline White & 157,846 & 0,000 & 4,089 & 0,129 & 105,267 & 0,000 & 31,632 & 0,000 \\
\hline BG & 14,473 & 0,000 & 0,251 & 0,616 & 13,519 & 0,000 & 1,184 & 0,276 \\
\hline Jarque-Bera & $4.561,601$ & 0,000 & 7,661 & 0,021 & 499,524 & 0,000 & 285,061 & 0,000 \\
\hline $\mathrm{N}$ & 4.571 & & 175 & & 2.610 & & 1.131 & \\
\hline
\end{tabular}

Nota. ${ }^{a}$ Corrigido por White; ${ }^{\mathrm{b}}$ De acordo com o teorema do limite central, o pressuposto da normalidade foi relaxado; ${ }^{\mathrm{c}}$ Corrigido por Newey-West. 
Tabela 7

Persistência dos Fluxos de Caixa Operacionais

\begin{tabular}{lcccccccc} 
& \multicolumn{1}{c}{ Completa $^{\mathbf{a}, \mathbf{b}}$} & \multicolumn{3}{c}{ Crescimento $^{\mathbf{b}}$} & \multicolumn{3}{c}{ Maturidade $^{\mathbf{c}, \mathbf{b}}$} & \multicolumn{3}{c}{ Declínio $^{\mathbf{c}, \mathbf{b}}$} \\
\cline { 2 - 9 } & coefic. & p-valor & coefic. & p-valor & coefic. & p-valor & coefic. & p-valor \\
\hline Constante & 0,045 & 0,000 & 0,079 & 0,000 & 0,056 & 0,000 & 0,040 & 0,000 \\
FCO $_{\text {it-1 }}$ & 0,381 & 0,000 & 0,174 & 0,020 & 0,388 & 0,000 & 0,361 & 0,000 \\
$\mathrm{R}^{2}$ ajustado & 0,158 & - & 0,025 & - & 0,175 & - & 0,140 & - \\
Teste F & 858,869 & 0,000 & 5,483 & 0,020 & 550,851 & 0,000 & 184,844 & 0,000 \\
White & 547,899 & 0,000 & 5,818 & 0,054 & 87,701 & 0,000 & 25,958 & 0,000 \\
BG & 7,594 & 0,005 & 0,000 & 0,977 & 8,842 & 0,002 & 4,118 & 0,042 \\
Jarque-Bera & 2150,334 & 0,000 & 22,658 & 0,000 & 271,321 & 0,000 & 102,013 & 0,000 \\
N & 4.549 & & 175 & & 2.587 & & 1.129 & \\
\hline
\end{tabular}

Nota. Na amostra Crescimento, a variável $\mathrm{FCO}_{\mathrm{it}-1}$ foi significativa ao nível de $10 \%$ (p-valor = 0,099), pelo MMG.

${ }^{a}$ Corrigido por White; ${ }^{b}$ De acordo com o teorema do limite central, o pressuposto da normalidade foi relaxado; ${ }^{\mathrm{c}}$ Corrigido por Newey-West.

Já as empresas em estágio de crescimento têm lucros mais persistentes do que as empresas em estágio de declínio, o que demonstra maior qualidade das informações financeiras e melhores inputs para o processo valuation, conforme foi pressuposto inicialmente, uma vez que essas empresas precisam atrair investidores para financiar seus projetos com VPL positivo (Grullon \& Michaely, 2004).

Como as empresas analisadas negociam seus títulos em bolsas de valores (algumas em mercados de capitais mais desenvolvidos que o brasileiro, inclusive com maior nível de exigência quanto à qualidade das informações) e têm a obrigatoriedade de utilizar os princípios contábeis geralmente aceitos, além de serem auditadas, pressupõe-se que o princípio da competência é utilizado de forma correta, de modo que os lucros serão melhores medidas de desempenho do que os fluxos de caixa (Dechow \& Schrand, 2004).

Na média do mercado (amostra completa) isso é comprovado, visto que a diferença entre a persistência dos lucros e dos fluxos de caixa é de 0,242 (0,623-0,381). Observa-se que individualmente também há a superioridade dos lucros operacionais em relação aos fluxos de caixa operacionais, porém as empresas em estágio de crescimento têm lucros $0,422(0,596-0,174)$ mais persistentes do que os fluxos de caixa, enquanto que as empresas em estágio de maturidade e declínio têm a seguinte diferença, respectivamente: $0,217(0,605-0,388)$ e $0,224(0,585-0,361)$.

Esses resultados podem ser justificados pelo fato de que firmas em crescimento têm necessidade de aumentar o capital de giro, por conta do crescimento das contas operacionais, em especial, clientes e estoques (E. L. Black, 1998; Grullon \& Michaely, 2004; Jenkins et al., 2004). Como a empresa em crescimento provavelmente terá aumento nos montantes alocados em clientes e estoques, por exemplo, o seu fluxo de caixa operacional tende a ser menor do que o das demais empresas e também mais volátil, dependendo dos períodos e dos projetos que estão sendo executados, o que implica em menor persistência do fluxo de caixa operacional.

Com base nos resultados apresentados, as hipóteses $2 \mathrm{a}$ e $2 \mathrm{~b}$ não podem ser rejeitadas, uma vez que os lucros são mais persistentes do que os fluxos de caixa, em todos os casos, e que os lucros das empresas em estágio de crescimento são mais persistentes do que os das empresas em fase de declínio. 


\section{Gerenciamento de resultados contábeis}

A última medida de qualidade das informações contábeis analisada neste trabalho é o comportamento oportunístico nos resultados, avaliada por meio do desvio-padrão dos accruals discricionários, estimados pelos resíduos das regressões pelo modelo de Pae (2005), não tabulados aqui.

Os resultados apresentados na Tabela 8 evidenciam que existem diferenças entre o gerenciamento de resultados das empresas alocadas nos três estágios do ciclo de vida, apontado que as empresas listadas no estágio de declínio gerenciam mais resultados do que as demais empresas, sendo as maduras as que menos gerenciam seus resultados. Porém, as evidências apontadas na Tabela 8 não podem ser consideradas estatisticamente diferentes, uma vez que o teste de Kruskal-Wallis indicou que não há diferenças significativas no gerenciamento de resultados das empresas alocadas nos diversos estágios do ciclo de vida.

Tabela 8

\section{Desvio-Padrão dos Accruals Discricionários}

\begin{tabular}{lcccccc}
\hline Estágio do Ciclo de Vida PAE & $\begin{array}{c}\text { Desvio- } \\
\text { padrão }\end{array}$ & $\begin{array}{c}\text { Qtd de } \\
\text { accruals } \\
\text { positivos }\end{array}$ & $\begin{array}{c}\text { Qtd de } \\
\text { accruals } \\
\text { negativos }\end{array}$ & $\begin{array}{c}\text { Levene } \\
\text { (p-valor) }\end{array}$ & $\begin{array}{c}\text { Kolmogorov } \\
\text {-Smirnov } \\
\text { (p-valor) }\end{array}$ & $\begin{array}{c}\text { Kruskal- } \\
\text { Wallis } \\
\text { (p-valor) }\end{array}$ \\
\hline Completa & 0,096 & 700 & 1.238 & - & - & - \\
Crescimento & 0,103 & 51 & 44 & - & - & - \\
Maturidade & 0,085 & 611 & 613 & - & - & - \\
Declínio & 0,119 & 253 & 267 & - & - & - \\
Testes & - & - & - & 15,344 & 0,070 & 0,318 \\
& & & & $(0,000)$ & $(0,000)$ & $(0,957)$ \\
\hline
\end{tabular}

Ainda de acordo com as evidências apontadas nesta seção, apesar de a dispersão dos accruals discricionários ter uma amplitude de 0,034 desvios-padrão do menor para o maior nível de gerenciamento de resultados, essa diferença não foi considerada estatisticamente significativa, o que faz com que a hipótese 3 seja rejeitada.

Além disso, avaliando a quantidade de observações de accruals discricionários positivos e negativos em cada caso, confirmou-se que a maioria dos accruals discricionários das empresas em crescimento tem viés positivo (Ball \& Shivakumar, 2008; Liao, 2008; Liu, 2008), o contrário nos outros casos (Dechow \& Ge, 2006; Grullon \& Michaely, 2004; Shyu \& Chen, 2009; Xu, 2007), ressaltando-se que as diferenças entre os grupos não foram significativas a $5 \%$.

\section{Considerações Finais}

Os resultados desta pesquisa sugerem que o ciclo de vida explica parcialmente o comportamento da qualidade das informações contábeis, pois foram encontradas evidências de que a qualidade das informações contábeis variou de acordo com os estágios de ciclo de vida das firmas brasileiras no período de 1995 a 2011.

Por meio da análise do conservadorismo, foi encontrado que os ativos operacionais líquidos das empresas maduras são mensurados de forma mais conservadora que as demais, e as empresas em estágio de crescimento são mais conservadoras que as empresas em estágio de declínio. Considerando os ativos operacionais líquidos na perspectiva do mercado, encontrou-se que as empresas no estágio de 
crescimento são mais conservadoras que as demais, seguidas das empresas no estágio de declínio, conforme era esperado, pelo modelo de Feltham e Ohlson (1995). Já quando se utilizou o modelo de Basu (1997), baseado no lucro líquido, as empresas em estágio de declínio são mais conservadoras que as demais, que são consideradas não viesadas quanto ao conservadorismo. Esse resultado foi diferente do que se esperava, contudo pode-se justificar porque que as empresas em estágio de declínio buscam minimizar o pagamento de dividendos, para manter os negócios em continuidade.

Em relação à persistência dos lucros e dos fluxos de caixa operacionais, observou-se que os lucros contábeis são mais persistentes do que os fluxos de caixa em todas as fases do ciclo de vida; sendo que os resultados na fase de maturidade são mais persistentes que nas demais fases, resultados estes similares aos apresentados nos trabalhos de Dechow e Schrand (2004) e Dickinson (2011). Também se encontraram evidências de que as empresas em estágio de crescimento têm lucros mais persistentes do que aquelas em estágio de declínio, corroborando os achados de Drake (2013), nos quais se evidenciou menor persistência nos resultados das empresas que estão na fase de declínio. Esses resultados sugerem que o ciclo de vida das firmas afeta a persistência dos lucros, devendo ser considerado na previsão dos resultados futuros e valuation.

Quando considerada a perspectiva do gerenciamento de resultados contábeis, apesar de ter sido encontrado que há um maior nível de discricionariedade nos resultados em empresas em estágio de declínio, essa diferença entre elas e as demais firmas não se apresenta estatisticamente significante.

Portanto, este trabalho apresenta evidências sobre alguns atributos da qualidade das informações contábeis e do ciclo de vida das firmas, corroborando as evidências de trabalhos anteriores em ambientes internacionais. Assim, verificou-se que existe relação entre a qualidade dos números contábeis e o ciclo de vida das firmas, destacando que tal comportamento tem implicações na avaliação de desempenho e do risco das empresas, bem como no processo de valuation, inclusive em um mercado emergente, como o Brasil.

Para trabalhos futuros, recomenda-se explorar algumas limitações deste trabalho, como segregar a amostra durante alguns períodos de tempo, como antes e após a adoção das normas internacionais de contabilidade, com um período de tempo maior, e antes, durante e após os períodos de crise, uma vez que esses eventos podem ter algum efeito sobre os resultados. Além disso, recomenda-se analisar os anos separadamente para analisar os efeitos individualmente, bem como explorar possíveis efeitos setoriais e suas possíveis interações com o ciclo de vida e a qualidade das informações contábeis.

\section{Referências}

Aharony, J. H. F., \& Yehuda, N. (2006). Corporate life cycle and the value relevance of cash flow versus accrual financial information [Working Paper $\mathrm{n}^{\circ}$ 34]. School of Economics and Management, Bolzano, Trentino-Alto Ádige, Italy.

Ahmed, A., Morton, R., \& Schaefer, T. (2000). Accounting conservatism and the valuation of accounting numbers: evidence on the Feltham-Ohlson (1996) model. Journal of Accounting Auditing and Finance, 15(3), 271-292. doi: 10.1177/0148558X0001500305

Alali F. A., \& Foote, P. S. (2012). The value relevance of international financial reporting standards: empirical evidence in an emerging market. The International Journal of Accounting, 47(1), 85108. doi: 10.1016/j.intacc.2011.12.005

Anthony, J., \& Ramesh, K. (1992). Association between accounting performance measures and stock returns. Journal of Accounting and Economic, 15(2/3), 203-227. doi: 10.1016/01654101(92)90018-W 
Ball, R., \& Shivakumar, L. (2008). Earnings quality at initial public offerings. Journal of Accounting and Economics, 45(2), 324-349. doi: 10.1016/j.jacceco.2007.12.001

Basu, S. (1997). The conservatism principle and the asymmetric timeliness of earnings. Journal of Accounting and Economics, 24(1), 3-37. doi: 10.1016/S0165-4101(97)00014-1

Beyer, A., Cohen, D. A., Lyz, T. Z., \& Walther, B. R. (2010). The financial reporting environment: review of the recent literature. Journal of Accounting and Economics, 50(2/3), 296-343. doi: 10.1016/j.jacceco.2010.10.003

Black, B. S., Carvalho, A. G., \& Gorga, É. (2012). What matters and for which firms for corporate governance in emerging markets? evidence from Brazil (and other BRIK countries). Journal of Corporate Finance, 18(4), 934-952. doi: 10.1016/j.jcorpfin.2011.10.001

Black, E. L. (1998). Life-cycle impacts on the incremental value-relevance of earnings and cash flow measures. Journal of Financial Statement Analysis, 4, 40-56.

Chiang, S., Lee, P., \& Anandarajan, A. (2011, August). The influence of corporate governance on innovative success: a life cycle analysis. Proceedings of the American Accounting Association's Annual Meeting, Denver, CO, USA.

Coulton, J. J., \& Ruddock, C. (2011). Corporate payout policy in Australia and a test of the life cycle theory. Accounting \& Finance, 51(2), 381-407. doi: 10.1111/j.1467-629X.2010.00356.x

Dechow, P. M., \& Ge, W. (2006). The persistence of earnings and cash flows and the role of special items: implications for the accrual anomaly. Review of Accounting Studies, 11(2/3), 253-296. doi: $10.1007 / \mathrm{s} 11142-006-9004-1$

Dechow, P. M., Ge, W., \& Schrand, C. (2010). Understanding earnings quality: a review of the proxies, their determinants and their consequences. Journal of Accounting and Economics, 50(1/2), 344401. doi: 10.1016/j.jacceco.2010.09.001

Dechow, P. M., \& Schrand, C. M. (2004). Earnings quality. Charlottesville: CFA Institute.

Dichev, I. D., Graham, J. R., Harvey, C. R., \& Rajgopal, S. (2013). Earnings quality: evidence from the field. Journal of Accounting and Economics, 56(2/3), 1-33. doi: 10.1016/j.jacceco.2013.05.004

Dickinson, V. (2011). Cash flow patterns as a proxy for firm life cycle. The Accounting Review, 86(6), 1969-1994. doi: 10.2308/accr-10130

Drake, K. (2013, February). Does firm life cycle explain the relation between book-tax differences and earnings persistence? Proceedings of the American Taxation Association Midyear Meeting: Research Forum. San Diego, CA, USA, 25.

Feltham, G. A., \& Ohlson, J. A. (1995). Valuation and clean surplus accounting for operating and financial activities. Contemporary Accounting Research, 11(2), 689-732. doi: 10.1111/j.19113846.1995.tb00462.x

Fraletti, P. B. (2004). Ensaios sobre taxas de juros em reais e sua aplicação na análise financeira (Tese de doutorado). Universidade de São Paulo, São Paulo, SP, Brasil.

Grullon, G., \& Michaely, R. (2004). The information content of share repurchase programs. The Journal of Finance, 59(2), 651-680. doi: 10.1111/j.1540-6261.2004.00645.x

Jenkins, D., Kane, G., \& Velury, U. (2004). The impact of the corporate life-cycle on the value-relevance of disaggregated earnings components. Review of Accounting and Finance, 3(4), 5-20. 
Lev, B., Sarath, B., \& Sougiannis, T. (2005). R\&D Reporting biases and their consequences. Contemporary Accounting Research, 22(4), 977-1026. doi: 10.1506/7XMH-QQ74-L6GG-CJRX

Liao, C.-N. (2008). Incentive reward control: based on the competitive advantage, transaction cost economics and organizational life cycle viewpoint. Human Systems Management, 27(2), 123-130. doi: 10.3233/HSM-2008-0673

Liu, M. (2008). Accruals and managerial operating decisions over the firm life cycle (Doctoral dissertation). Massachusetts Institute of Technology, Cambridge, MA, USA.

Lopes, A. B., Sant'Anna, D. P., \& Costa, F. M. (2007). A relevância das informações contábeis na Bovespa a partir do arcabouço teórico de Ohlson: avaliação dos modelos de Residual Income Valuation e Abnormal Earnings Growth. Revista de Administração da USP, 42(4), 497-510.

Marshall, A. (1890). Principles of economics: an introductory volume (8th ed.). London: McMillan and Co., Ltd.

Miller, D., \& Friesen, P. H. (1984). A longitudinal study of the corporate life cycle. Management Science, 30(10), 1161-1183. doi: 10.1287/mnsc.30.10.1161

Mueller, D. C. (1972). A life cycle theory of the firm. Journal of Industrial Economics, 20(3), 199- 219. doi: $10.2307 / 2098055$

Ohlson, J. A. (1995). Earnings, book values and dividends in equity valuation. Contemporary Accounting Research, 11(2), 661-687. doi: 10.1111/j.1911-3846.1995.tb00461.x

Ou, J. A., \& Penman, S. H. (1989). Financial statement analysis and the prediction of stock returns. Journal of Accounting and Economics, 11(4), 295-329. doi: 10.1016/0165-4101(89)90017-7

Pae, J. (2005). Expected accrual models: the impact of operating cash flows and reversals of accruals. Review of Quantitative Finance and Accounting, 24(1), 5-22. doi: 10.1007/s11156-005-5324-7

Park, Y., \& Chen, K. H. (2006). The effect of accounting conservatism and life-cycle stages on firm valuation. Journal of Applied Business Research, 22(3), 75-92.

Paulo, E., Antunes, M. T. P., \& Formigoni, H. (2008). Conservadorismo contábil nas companhias abertas e fechadas brasileiras. Revista de Administração de Empresas, 48(3), 46-60. doi: 10.1590/S0034-75902008000300005

Paulo, E., Cavalcante, P. R. N., \& Melo, I. I. S. L. de (2012). Qualidade das informações contábeis na oferta pública de ações e debêntures pelas companhias abertas brasileiras. Brazilian Business Review, 9(1), 1-26. doi: 10.15728/bbr.2012.9.1.1

Peasnell, K. V., Pope, P. F., \& Young, S. (2000). Detecting earnings management using cross-sectional abnormal accruals models. Accounting and Business Research, 30(4), 313-326. doi: $10.1080 / 00014788.2000 .9728949$

Penman, S. H., \& Zhang, X.-J. (2002). Accounting conservatism, the quality of earnings, and stock returns. The Accounting Review, 77(2), 237-264. doi: 10.2308/accr.2002.77.2.237

Ramaswamy, V., Ueng, J. C., \& Carl, L. (2008). Corporate governance characteristics of growth companies: an empirical study. Academy of Strategic Management Journal, 7(1), 21-33.

Richardson, S., Tuna, I., \& Wysocki, P. (2010). Accounting anomalies and fundamental analysis: a review of recent research advances. Journal of Accounting and Economics, 50(2/3), 410-454. doi: 10.1016/j.jacceco.2010.09.008 
Riedl, E. J. (2004). An examination of long-lived asset impairments. The Accounting Review, 79(3), 823-852.

Samadiyan, B., \& Rezaei, F. (2012). Investigating the relationship between stock prices and earnings quality using Leuz Parton-Simko and Penman models in firm's life cycle stages. Journal of Basic and Applied Scientific Research, 2(3), 2312-2324.

Shyu, J., \& Chen, Y. (2009). Diversification, performance, and the corporate life cycle. Emerging Markets Finance and Trade, 45(6), 57-6. doi: 10.2753/REE1540-496X450604

Teoh, S. H., Welch, I., \& Wong, T. J. (1998). Earnings management and the long-run market performance of initial public offerings. The Journal of Finance, 53(6), 1935-1974. doi: $10.1111 / 0022-1082.00079$

Teoh, S. H., Wong, T. J., \& Rao, G. R. (1998). Are accruals during initial public offerings opportunistic? Review of Accounting Studies, 3(1/2), 175-208. doi: 10.1023/A:1009688619882

Watts, R. L. (2003a). Conservatism in accounting part I: explanations and implications. Accounting Horizons, 17(3), 207-221. doi: 10.2308/acch.2003.17.3.207

Watts, R. L. (2003b). Conservatism in accounting part II: evidence and research opportunities. Accounting Horizons, 17(4), 287-301. doi: 10.2308/acch.2003.17.4.287

$\mathrm{Xu}$, B. (2007). Life cycle effect on the value relevance of common risk factors. Review of Accounting and Finance, 6(2), 162-175. doi: 10.1108/14757700710750838

\title{
Dados dos Autores
}

\author{
Ailza Silva de Lima \\ Jardim Cidade Universitária, Programa Multiinstitucional e Inter-regional de Pós-graduação em Ciências Contábeis, 58059- \\ 900, João Pessoa, PB, Brasil. E-mail: ailzalimma@gmail.com \\ Evelyne Vilhete Antonio de Carvalho \\ Jardim Cidade Universitária, Programa Multiinstitucional e Inter-regional de Pós-graduação em Ciências Contábeis, 58059- \\ 900, João Pessoa, PB, Brasil. E-mail: evellinevilhete@gmail.com \\ Edilson Paulo \\ Jardim Cidade Universitária, Programa Multiinstitucional e Inter-regional de Pós-graduação em Ciências Contábeis, 58059- \\ 900, João Pessoa, PB, Brasil. E-mail: e.paulo@uol.com.br \\ Luiz Felipe de Araújo Pontes Girão \\ Jardim Cidade Universitária, Programa Multiinstitucional e Inter-regional de Pós-graduação em Ciências Contábeis, 58059- \\ 900, João Pessoa, PB, Brasil. E-mail: luizfelipe @ ccsa.ufpb.br
}

